\title{
Erratum
}

13. Beerbaum P, Barth P, Kropf S et al (2009) Cardiac function by MRI in congenital heart disease impact of consensus training on interinstitutional variance. J Magn Reson Imaging 30:956-966

14. Sarikouch S, Kuehne T, Peters B et al (2010) Sexspecific pediatric percentiles for ventricular size and mass as reference values for cardiac MRI: assessment by steady-state free-precession and phase-contrast mri flow. Circ Cardiovasc Imaging 3:6576

15. Sarikouch S, Peters B, Mir TS et al (2009) Improved evaluation of postoperative tetralogy of Fallot by heart defect specific reference values: What is normal? Clin Res Cardiol 98:581-623

16. Bodhey NK, Beerbaum P, Sarikouch S et al (2008) Functional analysis of the components of the right ventricle in the setting of tetralogy of Fallot. Circ Cardiovasc Imaging 1:141-147

17. Gutberlet M, Spors B, Thoma T et al (2008) Suspected chronic myocarditis at cardiac MR: diagnostic accuracy and association with immunohistologically detected inflammation and viral persistence. Radiology 246:401-409

Kardiologe $2010 \cdot 4: 486-487$ - DOI 10.1007/s12181-010-0314-y

(C) Springer-Verlag 2010

\section{Moser ${ }^{1} \cdot$ T. Lickfeld $^{2} \cdot$ M. Möckel $^{3} \cdot$ P. Radke ${ }^{4} \cdot$ J. Vollert $^{3} \cdot$ Task Force} „Patientenpfade" der Deutschen Gesellschaft für Kardiologie Herz-Kreislaufforschung $\cdot$ C. Bode ${ }^{1}$

${ }^{1}$ Abteilung Innere Medizin III Kardiologie und Angiologie, Universitätsklinikum Freiburg

${ }^{2}$ Medizinische Klinik I, Städtische Kliniken Neuss - Lukaskrankenhaus- GmbH, Neuss

${ }^{3}$ Medizinische Klinik m. S. Kardiologie, Charité - Universitätsmedizin Berlin, Campus Virchow-Klinikum, Berlin

${ }^{4}$ Medizinische Klinik II (Kardiologie, Angiologie, Internistische Intensivmedizin), Universitätsklinikum Schleswig-Holstein, Campus Lübeck

\section{Erratum zu: „,Standard operating procedures“ zur Umsetzung der Leitlinien beim Nicht-ST-Hebungsinfarkt}

\author{
Kardiologe (2010) 4: 389-399 \\ http://dx.doi.org/10.1007/s12181-010-0284-0
}

Im oben genannten Beitrag wurde die

- Abb. 2 leider fehlerhaft wiedergegeben. Folgende Diagrammfelder sind betroffen:

- Rechtes Diagrammfeld knapp oberhalb des Zeitpunktes 60 min: Falsche Beschriftung: „V.a. NSTEMI/Troponin positiv“ Korrekte Beschriftung: „V.a. NSTEMI/Troponin negativ”.

- Rechtes Diagrammfeld zwischen den Zeitpunkten 60 min und $<72 \mathrm{~h}$ : Falsche Beschriftung: „Instabile AP/Troponin positiv“. Korrekte Beschriftung: „Instabile AP/Troponin negativ”.
Wir bitten um entsprechende Beachtung.

\section{Korrespondenzadresse}

Prof. Dr. M. Moser

Abteilung Innere Medizin III Kardiologie und Angiologie, Universitätsklinikum Freiburg Hugstetter Str. 55, 79106 Freiburg

Martin.Moser@uniklinik-freiburg.de 


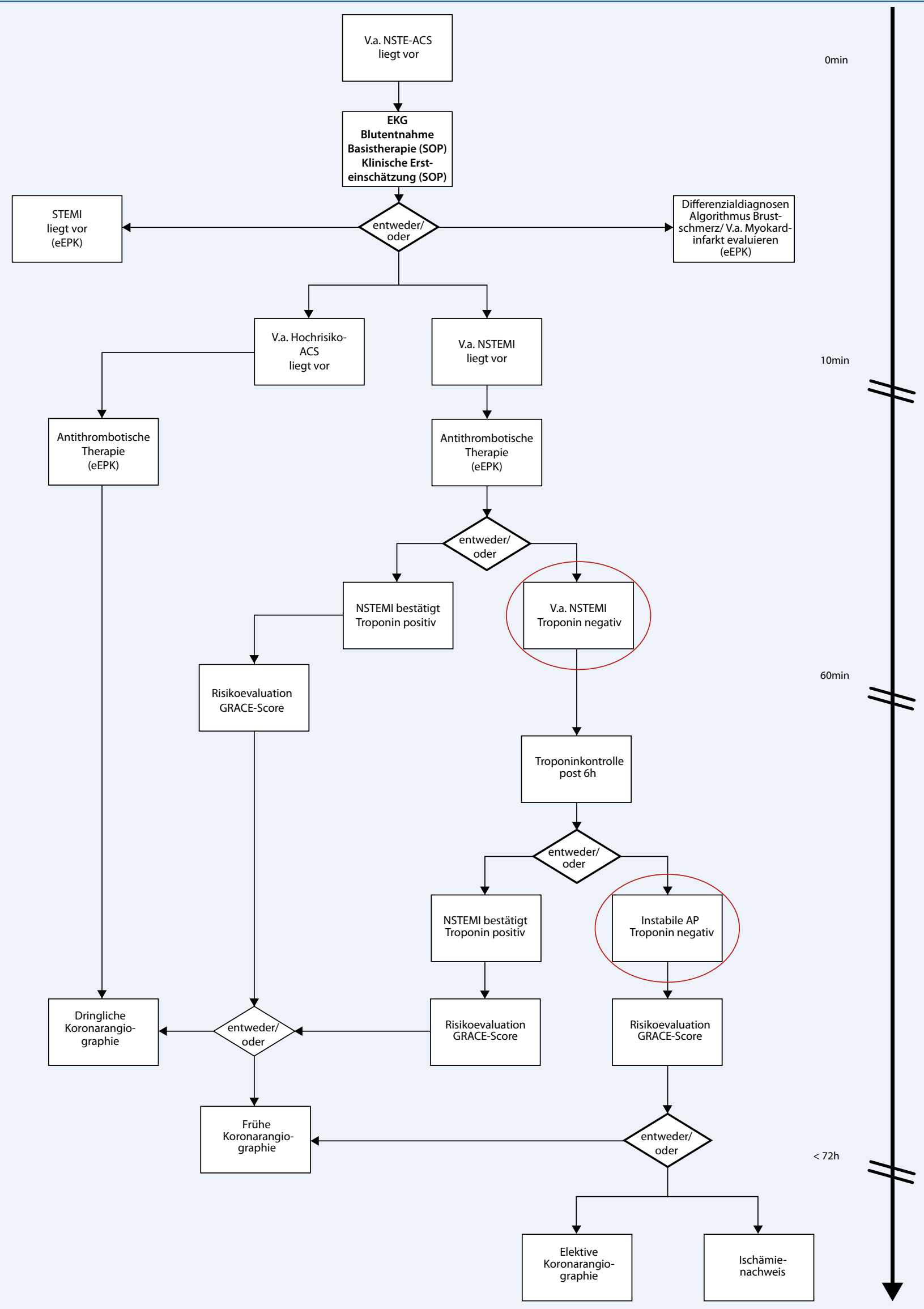

Abb. $2 \Delta$ Beispiel eines Flowcharts zum Ablauf bei Verdacht auf ein akutes Koronarsyndrom ohne ST-Elevation (NSTE-ACS) 